Cahiers « Mondes anciens »

ANCIENS

Histoire et anthropologie des mondes anciens

$6 \mid 2015$

Mères grecques

\title{
De mères à Grande Mère
}

Une relecture de la documentation archéologique en Sicile au regard des témoignages du Proche Orient

From Mothers to Great-Mother: A New Interpretation of the Archaeological

Documentation from Sicily with a Particular Attention to the Near East

\section{Giulia Pedrucci}

\section{(2) OpenEdition}

Journals

Édition électronique

URL : http://journals.openedition.org/mondesanciens/1366

DOI : 10.4000/mondesanciens.1366

ISSN : 2107-0199

Éditeur

UMR 8210 Anthropologie et Histoire des Mondes Antiques

Référence électronique

Giulia Pedrucci, « De mères à Grande Mère », Cahiers « Mondes anciens » [En ligne], 6 | 2015, mis en ligne le 16 février 2015, consulté le 19 avril 2019. URL : http://journals.openedition.org/ mondesanciens/1366 ; DOI : 10.4000/mondesanciens.1366

Ce document a été généré automatiquement le 19 avril 2019

Les Cahiers "Mondes Anciens » sont mis à disposition selon les termes de la licence Creative Commons Attribution - Pas d'Utilisation Commerciale - Pas de Modification 4.0 International. 


\section{De mères à Grande Mère}

Une relecture de la documentation archéologique en Sicile au regard des témoignages du Proche Orient

From Mothers to Great-Mother: A New Interpretation of the Archaeological

Documentation from Sicily with a Particular Attention to the Near East

\section{Giulia Pedrucci}

\section{Introduction}

1 Le but de cet article est de tenter d'expliquer la présence en Sicile d'un nombre extraordinairement élevé de terres cuites de kourophoroi et kourotrophoi, un sujet assez rare dans le monde ancien (surtout dans la mère patrie grecque) ${ }^{1}$. Cependant, avant d'analyser en détail les données archéologiques, on a trouvé nécessaire de faire un excursus sur une caractéristique très significative de l'île : la Sicile, en effet, est considérée comme un lieu «religieusement » au féminin, caractérisé au début par l'existence d'une «Grande Mère » locale, et ensuite par le culte, presque exclusif, de Déméter et Korè. Par conséquent, on a commencé en mettant en question l'existence d'une « Grande Mère » en général et d'une «Grande Mère sicilienne » en particulier. Ce premier paragraphe a été aussi l'occasion de présenter une brève histoire des études sur l'île, non seulement sur la Grande Mère, mais aussi sur un sujet étroitement lié à elle : celui de la religion indigène. L'avertissement d'Angelo Brelich, qui croyait que l'on ne pouvait pas connaître les cultes indigènes, tout en ne niant pas leur vraisemblable existence, doit être correctement contextualisé au regard de l'époque dans laquelle il a été exprimé et devrait être considéré aujourd'hui à surmonter.

2 Comme on peut le voir en analysant les données archéologiques, en fait, plusieurs terres cuites présentent des caractéristiques indigènes. On pense qu'elles pourraient être liées à un culte local, mais pas pour une «Grande Mère", comme les auteurs modernes ont souvent voulu le croire, mais pour plusieurs mères, c'est-à-dire des figures féminines, anonymes, associées de plusieurs façons à la maternité, que les Grecs auraient appelé par 
des noms génériques, tels que paides, meteres, nymphes, mais surtout Déméter et Korè. Le fait que certaines terres cuites montrent aussi des traits orientaux pourrait suggérer l'existence d'une relation directe entre la Sicile et l'Asie Mineure, ou même l'origine proche-orientale de certaines populations qui se sont installées sur l'île avant l'arrivée des Grecs.

\section{La « Grande Mère sicilienne » et la religion « indigène »}

Dans les études d'histoire des religions, il existe un "mythe» persistant et tenace, encombrant mais inconsistant, séduisant autant qu'évanescent, presque aussi ancien que la discipline elle-même: le mythe de la "Grande Mère ». Qu'est-ce que cette "Grande Déesse ", cette Potnia méditerranéenne, divinité suprême et omnipotente, à l'origine androgénétique, dont les compétences embrassent tous les aspects de la vie, héritière d'une société matriarcale ${ }^{2}$, entourée de parèdres en position secondaire. Une lubie ou bien un roman ${ }^{3}$, un conte de fées ${ }^{4}$, ainsi que Luisa Banti déjà en 1941 et Moses Finley en 1971 ont défini son histoire exceptionnelle, son «mythe», dans lequel tous les dynamismes historiques s'annulent. «La religiosità preistorica non è interpretabile in chiave femminile più di quanto non lo siano i pantheon politeistici più tardi » $a$ également observé, plus ou moins dans la même période, Ileana Chirassi Colombo ${ }^{5}$. chercheurs comme un mythe moderne à dépasser (ou déjà dépassé), il n'en finit pas de mourir et, souvent d'une manière souterraine, il remonte à la surface avec une persévérance obstinée. À ce propos, le cas de la Sicile est emblématique.

5 L'île est « victime » aussi d'un autre «mythe », qui trouve sa légitimation dans les sources anciennes, être l'île des Deux Déesses. Les auteurs anciens ne semblent pas avoir de doutes : la Sicile est par excellence la terre de Déméter et Korè ${ }^{6}$. La plupart des auteurs modernes non plus'. Cependant, sans vouloir nier l'auctoritas des auteurs anciens, on préfère vérifier le bien-fondé de cet "axiome", de ce "dogme», pour utiliser la définition d'Angelo Brelich ${ }^{8}$.

6 La documentation littéraire semble bien confirmée par l'archéologie : en effet, on trouve sur l'île beaucoup de statuettes de femmes (assises sur un trône, debout, au porcelet, à la parure, au polos, aux torches, aux enfants, etc.) ${ }^{9}$ dans des sanctuaires très différents, généralement associés à Déméter et Korè ou aux supposées «divinités chtoniennes ${ }^{10}$ " (l'expression chthonioi theoi «francamente, lascia un po'perplessi ! $\left.{ }^{11} »\right)$. Il y a tant de questions ouvertes sur ce sujet, qu'on ne peut pas les approfondir toutes $\mathrm{ici}^{12}$; on voudrait seulement insister sur le fait que le rapport entre le nombre de sanctuaires normalement attribués à Déméter et Korè et le nombre de sanctuaires attribués aux autres divinités en Sicile donne à penser qu'il faudrait réviser au cas par cas une bonne partie des attributions traditionnelles ${ }^{13}$.

7 La convergence en Sicile de ces deux puissants «mythes » a donné naissance à un troisième "mythe » : celui de la "Grande Mère sicilienne », nourri par et nourrissant l'idée que l'île est par excellence un pays de déesses à l'époque classique, et un pays de saintes et de madones plus tard. Une île " au féminin », breviter.

$\mathrm{Si}$ on analyse le travail des principaux spécialistes d'histoire des religions $\mathrm{du} \mathrm{xx}^{\mathrm{e}}$ siècle en Sicile (Emanuele Ciaceri, Biagio Pace, Eugenio Manni, Giuseppe Martorana) ${ }^{14}$, on comprend que les origines de cette supposée « Grande Mère sicilienne » sont à rechercher 
surtout dans l'œuvre de Manni. À partir de cette divinité originaire, on serait arrivé en Sicile " come in altri paesi di sostrato mediterraneo, ad una triplicità di figure, dalla quale emerge talvolta, primo abbozzo di mito, la figura della Madre accompagnata dalle Figlie ${ }^{15}$ ». Selon Manni, les divinités locales avaient une nature une et triple (inférieure, terrestre, supérieure) et, en raison de cette nature, lî̂le aurait été partagée par les Grecs entre Korè (déesse inférieure), Athéna (déesse terrestre) et Artémis (déesse supérieure, dans son aspect lunaire de Séléné). Les Grecs auraient identifié la même déesse sicilienne d'origine méditerranéenne avec l'une de ces trois déesses selon les cas. Martorana a recueilli l'héritage de son maître, en le développant dans une direction chrétienne.

Grâce à l'œuvre de chercheurs comme Manni et Martorana, donc, au siècle dernier, certaines « vulgates » sur l'île (comme l'existence d'une « Grande Mère sicilienne », le fait que l'île a été entièrement consacrée aux Deux Déesses) sont nées, et certaines imprécisions ou certaines légèretés terminologiques ont été acceptées et divulguées, telle l'utilisation inexacte et arbitraire de l'adjectif "chtonien». Les porte-parole de ces «vulgates» n'ont pas été seulement des historiens des religions mais aussi des archéologues, qui ont vu des Déméter presque partout et ont proposé des interprétations discutables, comme celle très connue relative au thesmophorion de Bitalemi et à sa supposée continuité avec le culte marial ${ }^{16}$.

En parlant de croyances religieuses de populations non grecques, on ne peut pas éviter de mentionner le très célèbre (et sévère) avertissement d'Angelo Brelich: les populations indigènes de Sicile ont eu évidement leurs cultes, qui ont eu sûrement quelque influence sur la religion des colonisateurs grecs, mais il n'est pas possible pour nous de la/les reconstruire.

11 Nicola Cusumano a consacré un article très précieux sur l'intervention de Brelich ${ }^{17}$. Il considère la question à partir du climat culturel de ces années : en 1964, Manni venait de publier Sicilia pagana ${ }^{18}$ et, à l'occasion du Congresso internazionale di studi sulla Sicilia antica à Palerme, il voulait renforcer la ligne suivie dans le livre, où - comme on vient de le voir la notion de "substrat » dominait ${ }^{19}$ pour proposer un cadre à la religiosité insulaire caractérisée par la résurgence constante, des premiers établissements préhistoriques jusqu'à l'arrivée du christianisme, d'une "religiosité sicilienne » originaire, cristallisée autour de la figure d'une "Grande Mère ", avec un parèdre en position subalterne : un pattern qui, au-delà de quelques superficiels changements dans le nom, était capable de survivre toujours identique dans son aspect originel, en s'imposant aux gens nouveaux qui s'établissaient dans l'île. Le résultat le plus important de cette dynamique était la « sicilianisation » ou " indigénisation » des Grecs siciliens, dont la culture ne représentait que le produit de l'absorption du substrat: derrière les mythes et les cultes grecs, il était toujours possible de voir la contrepartie locale.

12 Le caractère contraire au sens de l'histoire du concept de "sicilianisation» ou «indigénisation» est bien démontré par le fait qu'on peut l'utiliser pour des buts opposés : la sicilianisation, en effet, peut être utilisée dans les deux directions, pour célébrer ou bien pour dévaluer la composante indigène, suivant qu'on veut la légitimer ou bien la condamner ${ }^{20}$.

13 L'inclination à chercher les origines, en utilisant souvent le passé pour comprendre le présent, soit positivement soit négativement, a, quand même, des racines bien plus lointaines. 

Emanuele Ciaceri, selon lequel les superstitions contemporaines peuvent « con cammino a ritroso $»^{21}$ contribuer à reconstruire les cultes indigènes. Il s'agit d'une approche qu'on trouve radicalisée dans l'œuvre de Biagio $P^{2}{ }^{22}$, qui pousse à des conséquences extrêmes le nationalisme déjà présent dans la pensée de Pais. La traditionnelle vision hellénocentrique basculait : c'est dans la culture indigène, et non dans la culture grecque, qu'on devait chercher les origines de l'Italie entière. de continuité avec la ligne Pais-Ciaceri-Pace, renforcée et exaspérée par la synergie avec le mythe du substrat méditerranéen, qui avait connu un développement parallèle ${ }^{23}$, même si Manni ne partageait pas avec ses prédécesseurs certaines idées nationalistes ou régionalistes. rien dans la documentation en notre possession ne nous autorise à parler d'« indigénisation de la grécité ». Même si on arrive à repérer des éléments indigènes dans la culture grecque, il s'agira de comprendre leurs fonctions dans le nouveau contexte historique, qui a conditionné le processus de «re-sémantisation» du culte tel qu'on le connait grâce aux sources grecques. Ce qu'on appelle l'« héritage indigène » a été élaboré et adapté à un nouveau système, fondé sur de nouveaux rapports sociaux qui conduisent à des recompositions identitaires ${ }^{25}$.

17 Il est toujours difficile de ne pas être d'accord avec l'enseignement de Brelich, mais, à notre avis, cela ne signifie pas renoncer a priori à essayer d'interpréter les données que nous avons aussi avec une clé non grecque. On n'oublie pas que les erreurs de demain sont toujours préférables à celles d'hier ${ }^{26}$, même si on ne dispose pas d'une base solide, parce que, pour citer une phrase très célèbre d'Edgar Morin, « la connaissance progresse en intégrant en elle l'incertitude, non en l'exorcisant ». Et, en effet, nous sommes persuadée qu'il faut regarder les populations non grecques pour essayer de comprendre la documentation archéologique dont on dispose, en ayant toujours un œil tourné vers l'Orient.

18 Entre ceux qui ont vu des « Grandes Déesses » indigènes partout et ceux qui ont toujours refusé le moindre essai de reconstruire des aspects de la religion locale, ne peut-il pas exister une troisième voie? Une troisième voie qui pourrait être empruntée même pour ce qui concerne les attributions à Déméter et Korè, en passant à travers une attentive révision au cas par cas.

\section{Kourotrophoi et kourophoroi}

19 On vient de relativiser le «mythe » de la "Grande Mère » en Sicile. Les "mythes ", cependant, trouvent généralement leurs raison dans la réalité. Comme Larissa Bonfante l'a observé dans les années quatre-vingts ${ }^{27}$, en Sicile, on trouve une quantité extraordinairement élevée, surtout si on la rapporte à celle de la Grèce continentale des époques archaïque et classique, de représentations (normalement indiquées comme $e x$ $v^{v}{ }^{28}{ }^{28}$, peut-être d'une manière un petit peu sommaire) de femmes aux enfants dans les bras ou sur les épaules (kourophoroi $i^{29}$ ) ou de femmes nourrissants un enfant (kourotrophoi ${ }^{30}$ ). Pour expliquer cette différence documentaire entre Sicile et Grèce, très significative et 
passionnante, surtout en cas d'allaitement, Bonfante a parlé avec justesse des différences culturelles et religieuses ; toutefois, il convient de réévaluer son argumentation.

Pour commencer, on présentera dans le détail la documentation en notre possession.

Malgré quelques possibles inexactitudes et quelques probables oublis, dans un précédent travail ${ }^{31}$, on a catalogué les matériaux suivants :

- Géla (acropole) : 2-3 kourotrophoi, nombreuses kourophoroi (deux aux colombes). Deuxième moitié du v $v^{\mathrm{e}}$ siècle av. J.-C. environ. 1 kourotrophos (?) style tardo-dédalique de la fin du vII siècle av. J.-C. En majorité des produits de l'artisanat local, mais dans un milieu déjà très hellénisé. Divinité : Athéna, peut-être avec Déméter (déjà à partir de la fin du vi ${ }^{\mathrm{e}}$ siècle av. J.C. ?) et Héra. Thesmophories.

- Bitalemi (position par rapport à Géla: extra-urbaine ${ }^{32}$ ): 3 (peut-être 4) kourotrophoi, nombreuses kourophoroi, 1 singe, 1 colombe. Le sanctuaire est fréquenté à partir du $v^{\mathrm{e}}$ siècle av. J.-C. Le type de la kourophoros debout ou assise avec un enfant dans les bras ou sur l'épaule est attesté seulement à partir de la moitié $d u v^{e}$ siècle av. J.-C. La facture des kourotrophoi est très pauvre et sommaire. Divinité : Déméter (Pediò ?). Thesmophories.

- Carrubazza (position par rapport à Géla : suburbaine) : 1 kourophoros, 1 statuette de femme enceinte. Divinités : Déméter et Korè.

- Fontana Calda (position par rapport à Géla : extra-urbaine) : 1 kourophoros, 2 statuettes de femme enceinte. Divinités: Déméter et Korè ou nymphes? Sanctuaire pour femmes enceintes, qui y faisaient des ablutions ?33 $^{33}$

- Monte Bubbonia (position par rapport à Géla : extra-urbaine, centre sicane) : 1 kourophoros (? ), 1 kourophoros. $\mathrm{VI}^{\mathrm{e}}$ siècle av. J.-C. Facture indigène. Divinité : ?

- Capodarso (position par rapport à Géla : extra-urbaine, centre sicane) : statuette de femme enceinte hydrophore.

- Agrigente: 2 kourotrophoi, nombreuses kourophoroi (quelques singes, un homme). Les kourophoroi sont datées vers la fin du $\mathrm{VI}^{\mathrm{e}}$-début $\mathrm{v}^{\mathrm{e}}$ siècle av. J.-C. Divinités : le sanctuaire est connu comme "sanctuaire de divinités chtoniennes », les divinités tutélaires sont Déméter et Korè, mais, étant donnés le nombre et l'hétérogénéité des sanctuaires présents dans la même zone, on peut penser qu'il y avait d'autres divinités. Thesmophories.

- Vassallaggi (position par rapport à Agrigente : extra-urbaine, centre sicane) : 1 kourophoros à la colombe (?). Environ 530 av. J.-C. Divinités : Déméter et Korè vénérées dans deux grottes, mais la statuette n'est pas contextualisée.

- Sélinonte: «Edificio Triolo nord»: 3 (+1 égyptisante +1 ?) kourotrophoi, nombreuses kourophoroi. Statuettes d'enfants emmaillotés. 1 statuette de femme au singe sur l'épaule. D'autres kourotrophoi, dont l'une pourrait être datée du viI ${ }^{\mathrm{e}}$ siècle av. J.-C. Une statuette de possible production locale. La colline de la Gaggera est fréquentée au moins à partir de la fin du VII ${ }^{\mathrm{e}}$ siècle av. J.-C. Divinité : Héra. Origine sicane du complexe de la Malophoros?

- Ségeste (Contrada Vanella): 1 kourophoros. Fin VI ${ }^{\mathrm{e}}$-début v $\mathrm{v}^{\mathrm{e}}$ siècle av. J.-C.

- Mégara Hyblaea : 2 kourotrophoi, 5 kourophoroi. Statue très célèbre de kourotrophos avec deux enfants, provenant d'une tombe. Produit local, 550 av. J.-C. environ. Lien très particulier entre les habitants de Mégare et les populations locales. Divinité : Hybla ? Origine sicane?

- Syracuse : 5 kourophoroi. Thesmophories?

- Héloros : 1 kourotrophos (?). Sanctuaire de Déméter et Korè

- Adrano (Syracuse) : 1 kourotrophos. Divinités : une culte de Déméter et Korè est attesté, mais la statuette n'est pas contextualisée.

- Camarina : 3 kourotrophoi, beaucoup de kourophoroi. Lien très particulier avec les populations locales (voir Mégara Hyblaea). Divinités : culte très important de Déméter et Korè, en 
particulier de Korè avec son époux Hadès, mais les statuettes ne sont pas contextualisées (sauf celles provenant de la nécropole de Passo Marinaro). Probable culte d'Artémis, culte d'Athéna (déesse poliade) et culte de la nymphe Camarina.

- Scornavacche (centre grec dépendant de Camarina): 1 kourotrophos, 1 kourophoros hellénistique (Aphrodite ?), quelques statuettes de femmes enceintes (?). Divinité : aucun culte particulier, (Scornavacche était un centre de production).

- Catane : 1 kourophoros, singes provenant du sanctuaire de Déméter. Thesmophories? Des Bouches de Simeto provient la soi-disant «Dea del Simeto ». Musée de Catane (provenance incertaine, peut-être Camarine) : 2 kourotrophoi, 7 kourophoroi. Toutes de la fin du $\mathrm{VI}^{\mathrm{e}}$ siècle av. J.-C., sauf une kourophoros début du ve siècle av. J.-C. 1 statuette d'enfant emmailloté.

- Grammichele (position par rapport à Leontinoi : sanctuaire extra-urbain, dans un centre sicule) : nombreuses kourophoroi. Statuettes d'enfants emmaillotés. Rapports très bons avec les populations locales. Divinité : sanctuaire de Déméter et Korè.

- Randazzo: 1 kourotrophos. Milieu du v siècle av. J.-C. Provenance : nécropole. Divinité :?

- Himère : 1 kourotrophos. Iv ${ }^{\mathrm{e}}$ siècle av. J.-C. Provenance : « Tempio della Vittoria ». Divinité : la divinité la plus importante était Athéna, mais il y avait aussi un culte en l'honneur de Déméter et Korè.

- Centuripe (centre sicule) : 2 kourotrophoi (1 Aphrodite) d'époque hellénistique, 2 kourophoroi. Statuettes d'enfants emmaillotés. Divinité : ? (voir Adrano).

- Lipari : 1 kourophoros ( ?), Aphrodite sur une vase de la fin du IV siècle av. J.-C. Statuettes d'enfants emmaillotés. Rapports très bons avec les populations locales. Divinités : Déméter et Korè?

- $\underline{\text { Sicile punique }}: 1$ kourotrophos (?), 1 statuette archaïque de femme qui se comprime les seins, 5 Isis lactans.

- Provenance incertaine $: 1$ statuette archaïque avec un enfant dans les bras ; 2 kourophoroi fin $\mathrm{VI}^{\mathrm{e}}$-début $\mathrm{V}^{\mathrm{e}}$ siècle av. J.-C. ; 1 statuette de femme assise sur un trône avec un enfant de la fin $\mathrm{du} \mathrm{VI}^{\mathrm{e}}$ siècle av. J.-C.; 1 statuette de femme à l'enfant et oiseau ; 1 statuette d'une femme avec un enfant à côté et une statue (Déméter) sur l'épaule gauche.

- Terravecchia di Cuti (centre indigène sous l'influence de Gela à partir des $\mathrm{VI}^{\mathrm{e}}-\mathrm{v}^{\mathrm{e}}$ siècle av. J.C.) : 8 poids de tisserand votifs, dont 6 avec peut-être des noms de divinités féminines (dont $\left.A k k a^{34}\right)$. Sanctuaire de Déméter et Korè? 


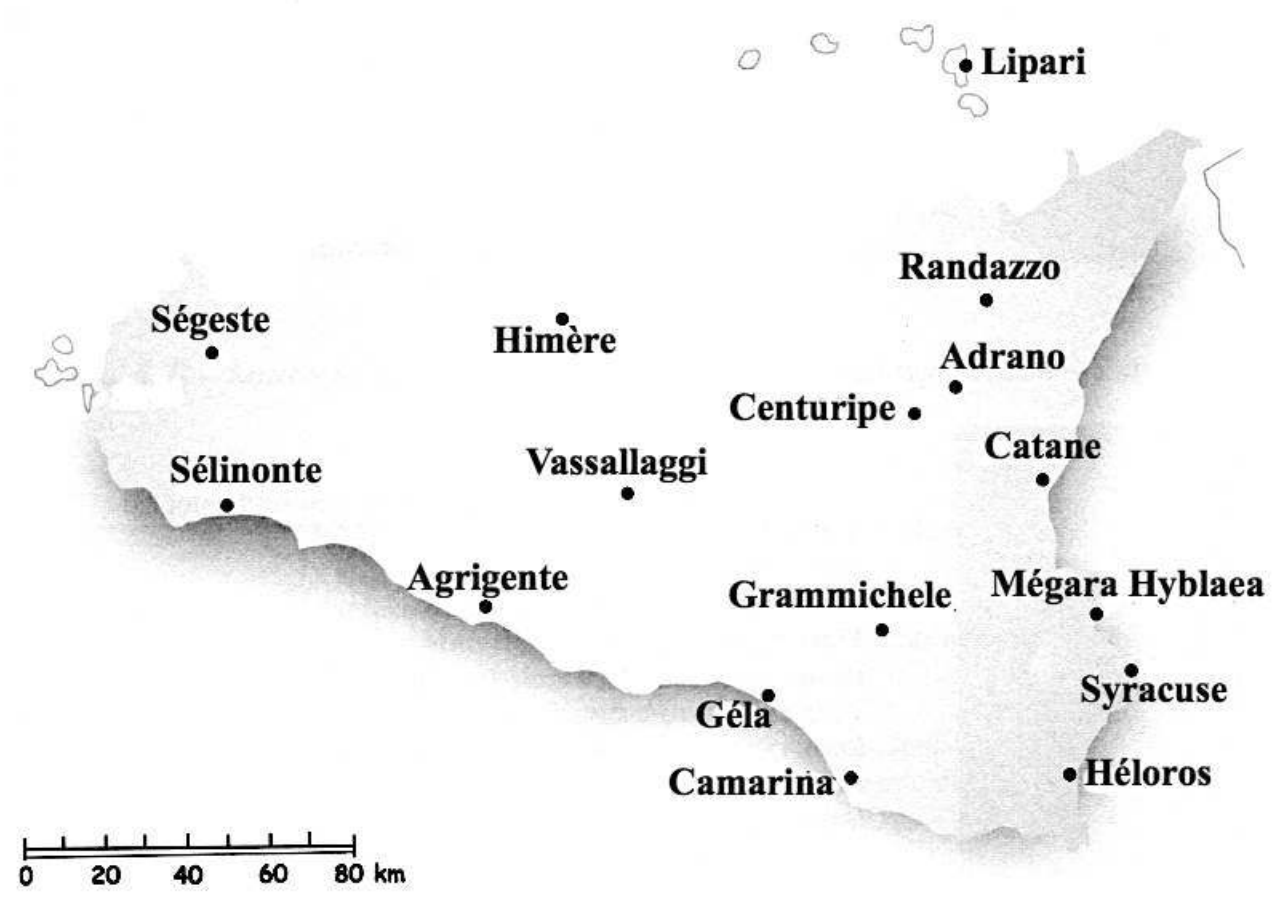

22 Je voudrais attirer l'attention en particulier sur cinq pièces parmi la vaste documentation qu'on vient de présenter. Il s'agit de quatre statuettes et d'une statue de probable production locale qui montrent des influences proche-orientales visibles. 
Fig. 1 : Kourotrophe (?) en terre cuite

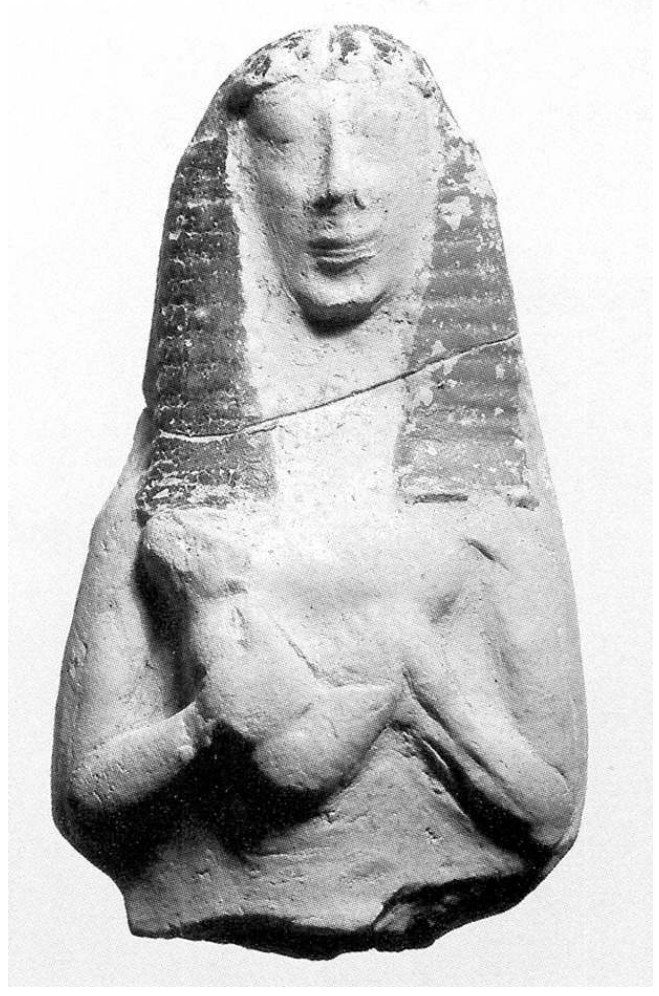

Caltanissetta, Museo Archeologico, inv. S. MAV 606 ; fin du VIle siècle. Pedrucci 2013a, p. 339, S. 96.

La première arrive de Géla et est très importante pour la datation et la facture : elle est de style tardo-dédalique, du dernier quart du viI ${ }^{\mathrm{e}}$ siècle av. J.-C. (la plus ancienne de l'ensemble), et de production locale. La statuette présente un visage allongé, un nez proéminent, des lèvres qui sourient, des boucles sur le front et des bandes horizontales sur le côté, les bras pliés pour soutenir un enfant appuyé sur le sein (Fig. 1) ${ }^{35}$. 
Fig. 2 : Kourotrophe (?) en terre cuite

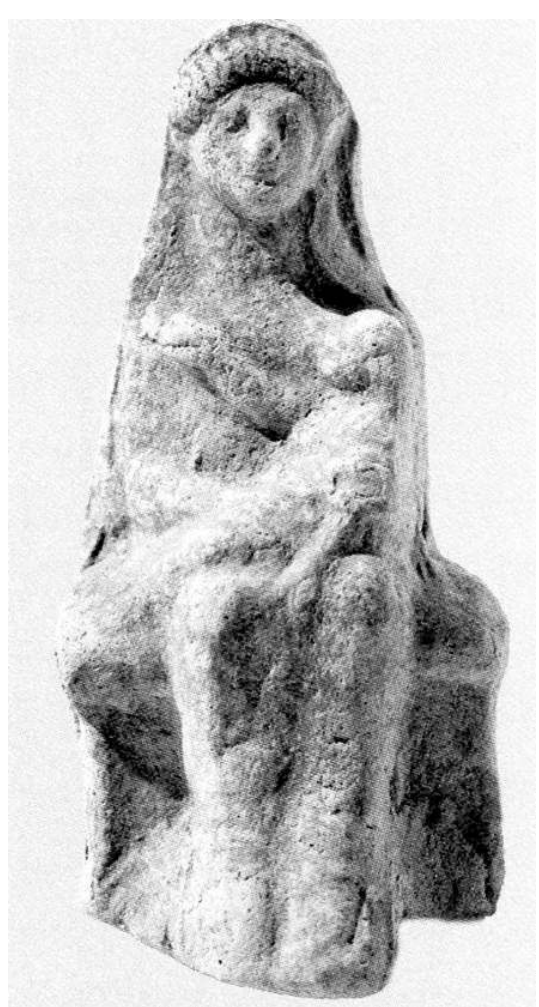

Caltanissetta, Museo Archeologico, inv. M.B.35465 ; fin du Vle siècle. Pedrucci 2013a, p. 340, S. 97. 


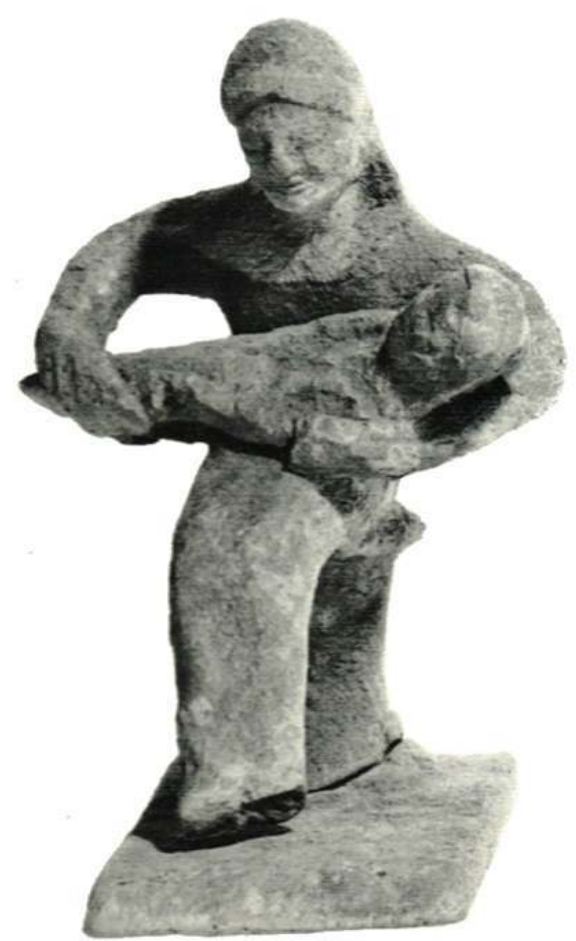

Syracuse, Museo Archeologico Regionale P. Orsi, inv. n. 24905 ; Vle siècle. Pedrucci 2013a, p. 340, S. 99

De Monte Bubbonia (toujours près de Géla) proviennent, d'une part, une statuette représentant une femme qui porte un enfant dans le bras, datée du dernier quart du $\mathrm{VI}^{\mathrm{e}}$ siècle av. J.-C (Fig. 2), dont la main droite est tendue vers le sein (il s'agit à vrai dire d'un type très fréquent en Sicile) ${ }^{36}$ et, d'autre part, une statuette représentant une femme assise, qui porte un enfant sur les genoux datant $\mathrm{du} \mathrm{VI}^{\mathrm{e}}$ siècle av. J.-C., très intéressante parce que les archéologues n'hésitent pas à la définir comme de facture indigène (Fig. 3) ${ }^{37}$. La première, une kourophoros peut-être plutôt qu'une kourotrophos (même si le geste de la main vers le sein est très significatif), provenant d'une tombe, a une moue qui ressemble à une sourire archaïque et un visage aux traits grossiers, presque "clownesque», qui pourraient faire penser à une production locale. Toutefois, si l'on considère les analogies avec les statuettes présentant le même geste et les mêmes traits stylistiques qui renvoient au milieu rhodo-crétois, il est plus vraisemblable de penser à un travail grossier, de facture indigène car, comme on l'a déjà souligné, à Monte Bubbonia, les Grecs et les non Grecs vivaient ensemble pacifiquement dès l'époque archaïque. 
Fig. 4 : Kourophore en terre cuite

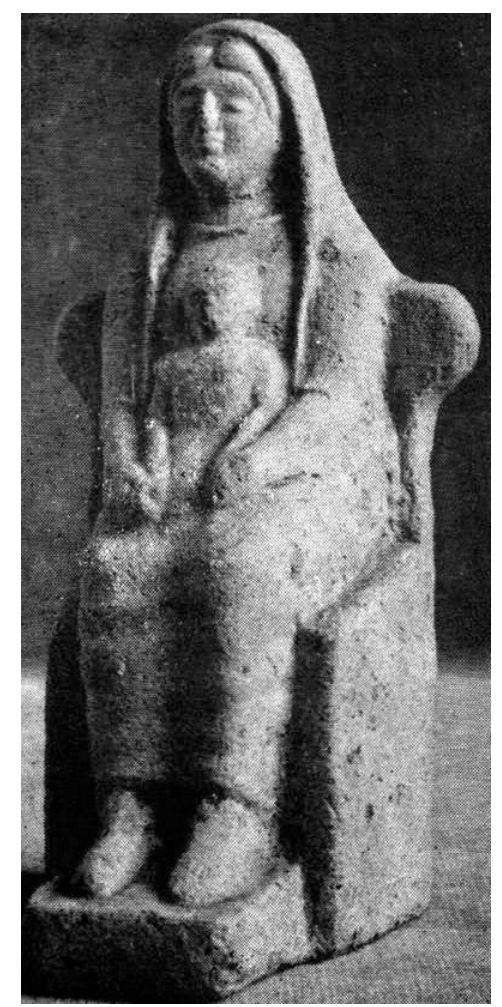

Musée du Louvre, Paris, inv. n. CA 2944 ; vers 500 a.C. Pedrucci 2013a, p. 341, S. 100.

Il faut également faire une place à une terre cuite du Musée du Louvre qui présente une facture très particulière et une posture insolite : la femme, assise en trône, a un corps bien plus trapu que les autres statuettes et les traits du visage aussi sont différents. Les cheveux sont divisés au centre, le rendu est très minutieux, comme celui du voile qui arrive jusqu'aux coudes et de la robe. Les jambes reposent sur un pose-pieds et sont séparées, la robe arrive jusqu'aux pieds. Il y a un détail très surprenant et délicat: les mains de la femme serrent celles de l'enfant, assis sur elle en position frontale. On ne connait pas d'exemplaires analogues. Les mains de la femme et le bras de l'enfant sont très élaborés. Cette composition semble suggérer que les deux sont liés par un sentiment très profond, mais, en même temps, l'artiste a voulu souligner l'individualité de l'enfant, en séparant son corps de celui de la femme (de la mère ?). On peut supposer qu'il s'agit d'un artefact local, malgré quelques influences ioniennes (Fig. 4) ${ }^{38}$. 
Fig. 5 : Statue de kourotrophe en pierre

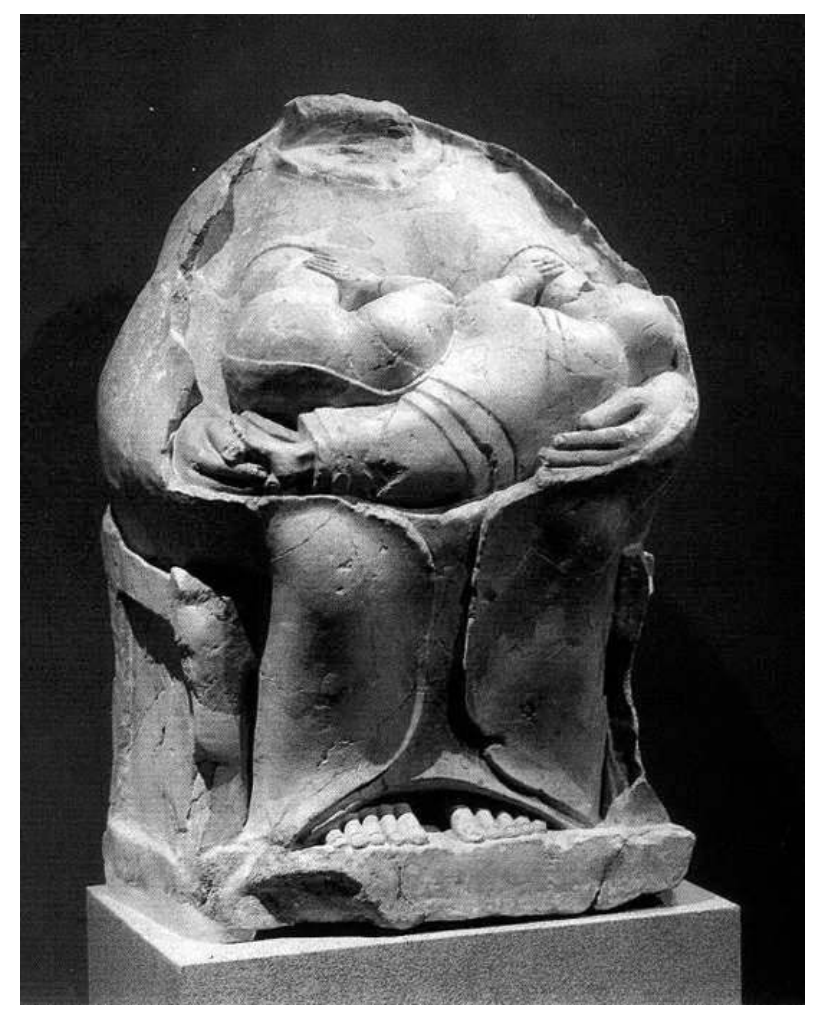

Syracuse, Museo Archeologico Regionale P. Orsi, inv. n. 53234 ; vers 550 a.C. Pedrucci 2013a, p. 340, S. 198.

En dernier lieu, on parlera de la célèbre kourotrophos en train d'allaiter deux enfants de Mégara Hyblaea (fig. 5) ${ }^{39}$. Cette statue (il ne faut pas oublier qu'il s'agit d'une vraie statue, pas d'une statuette) présente une évidente influence locale. Elle provient de la nécropole septentrionale. Elle est acéphale, vêtue d'un chiton et entièrement enveloppée d'un manteau lourd, qui lui couvre les épaules en laissant découverts le sein et le ventre, en dessinant une espèce de coquillage, dans lequel deux enfants emmaillotés se croisent. On est frappé par le réalisme (très évident si l'on considère les enfants attachés aux mamelons, tenant le sein de la femme qui sort de la robe par des ouvertures) associé à la stylisation du manteau et à un détail : l'ouverture courbée pour les pieds. Ce motif est typique de la statuaire proche-orientale, en particulier des courbures de ce type-ci sont fréquentes en Asie Mineure, par exemple sur la soi-disant Prêtresse d'Éphèse (570 av. J.C. environ) de l'Héraion de Samos (570-560 av. J.-C. environ), sur une statue d'homme assis de Didymes (première moitié $d u \mathrm{VI}^{\mathrm{e}}$ siècle av. J.-C.) et dans la soi-disant Dame d'Auxerre (moitié du vII ${ }^{\mathrm{e}}$ siècle av. J.-C.) ${ }^{40}$. La construction volumétrique présente des caractéristiques typiques de la statuaire indigène, visibles, par exemple, aussi dans une statue de femme assise de Grammichele (d'où provient aussi une tête au heaume, avec les mêmes traits nets et durs de la kourotrophos ${ }^{41}$ ).

La nudité complète, cependant, selon Vincenzo La Rosa, n'est pas typique de l'art indigène, tandis qu'elle l'est des statuettes dédaliques. En Sicile on connait un seul parallèle, à savoir la soi-disant «Dea del Simeto » qui, avec la kourotrophos de Mégara Hyblaea, représente un exemplaire très rare de grande statuaire. Cette statue aux seins 
débordants, qui pourrait remonter à la première moitié $d u$ ve siècle av. J.-C., ne ressemble pas à la production grecque ${ }^{42}$. vraisemblablement des conséquences non seulement sur le vie religieuse de Mégara Hyblaea, mais aussi sur celle de sa colonie principale, Sélinonte, qui se distingue des autres cités siciliennes pour sa position de confin ${ }^{51}$. En effet, la proximité de colonie avec la Sicile punique, des Sicanes et même des Sicules (étant donnée la position de la patrie d'origine), où les rapports entre populations différentes étaient au moins de bon voisinage, pourrait expliquer que s'y trouve le nombre en valeur absolue le plus élevé de kourotrophoi et kourophoroi, auxquelles il faut ajouter quelques statuettes d'enfants emmaillotés. Bien que dans le soi-disant «Edificio Triolo nord» (d'où la plupart des pièces proviennent) on ait mis au jour une probable dédicace pour Héra, la divinité principale de l'aire est une déesse évoquée tout simplement comme malophoros, porteuse de fruits ${ }^{52}$. Géla, si l'on considère toute sa chôra, surtout Bitalemi, où il y a un sanctuaire soi-disant extra-urbain (car particulièrement loin de la cité) dédié à une divinité maternelle et chtonienne, selon les définitions que nous avons proposées, vraisemblablement interprétée par les Grecs comme Déméter, peut-être associée à Korè. À Bitalemi, on trouve mentionnée une autre divinité probablement non grecque, Pediò, mais on ne peut rien dire de plus spécifique sur elle.

En ce qui concerne Gela, comme pour Agrigente et Lentinoi (cité-mère de Grammichele), il est très intéressant de souligner que, même si dans les sources on ne trouve pas de références à l'ouverture vers les populations non grecques, la capillarité de la pénétration dans le territoire fait supposer qu'existaient des rapports non conflictuels (en revanche, la zone où les relations entre Grecs et non Grecs étaient moins fortes est la zone 
corinthienne $\left.{ }^{53}\right)$. À cet égard, une statuette tardo-dédalique de la fin $\mathrm{du} \mathrm{VII}^{\mathrm{e}}$ siècle représente un exemplaire très intéressant (voir Panvini et Sole 2009, II, p. 77, VII, p. 19 ; Pedrucci 2013a, feuillet 96).

En troisième place, il y a Camarine, même si les témoignages qui proviennent de la cité sont probablement plus nombreux que l'on peut le penser. La caractéristique la plus significative de Camarine est la même que celle de Mégara Hyblaea et Sélinonte : le lien très fort avec les populations non grecques. Bitalemi aussi, étant données la localisation et la typologie du culte, se distinguait vraisemblablement par des rapports de bon voisinage avec les non Grecs.

Des sites périphériques provient la plupart des autres kourotrophoi : Himère, Adrano, Randazzo, Scornavacche. Si l'on excepte la terre cuite provenant d'Agrigente, les kourotrophoi retrouvées sur des acropoles sont très rares. À titre de confirmation supplémentaire pour le cadre qu'on est en train de dessiner, on rappellera le nombre élevé des kourophoroi et des enfants emmaillotés retrouvés dans des sanctuaires extraurbains, en plus de ceux déjà mentionnés: Monte Bubbonia, Vassallaggi, Centuripe, Grammichele. Un nombre considérable de ces centres étaient habités par des populations sicules ou sicanes. Les statuettes sont souvent des produits de l'artisanat local ou montrent des évidentes influences de l'artisanat local. Si l'on prend en considération la très pauvre documentation écrite, on découvre que le culte des divinités féminines, probablement liées à la maternité, comme Anna, Akka, les paides, les meteres, est typique de zones rurales et périphériques.

\section{De mères à « Grande Mère »}

Il faut évidemment expliquer une documentation si riche et singulière. Il existait vraisemblablement en Sicile le culte des plusieurs mères (avec une minuscule et au pluriel ${ }^{54}$ ), c'est-à-dire des figures féminines, anonymes, dont nous parlent les sources classiques et dont on a des témoignages archéologiques. On fait normalement remonter leur culte, de façon évidemment approximative, à l'époque archaïque.

On veut parler en particulier des nymphes, des meteres, d'Anna et des paides, mais aussi d'une série de divinités secondaires qui ont contribué, avec le temps, à faire de la Sicile une terre au féminin sur le plan religieux, comme on vient de le voir. Ces figures divines aux yeux des Grecs peu différenciées, avec des caractéristiques et des origines très incertaines, se trouvent à côté des plus célèbres divinités importées de la Grèce, dotées d'une identité bien définie (dont on souligne souvent le faible instinct « maternel »55).

À côté de ces divinités, on doit ajouter une série de figures féminines à l'enfant (pas nécessairement leur mère) représentées dans plusieurs terres cuites, que rien ne nous autorise à classifier comme déesses.

Une mise au point serait nécessaire: la pauvre, vieille, et quelques fois imprécise bibliographie dont on dispose sur les sanctuaires "secondaires" et le défaut de localisation exacte pour un nombre considérable de pièces nous obligent à affirmer que les statuettes semblent être archaïques, qu'elles semblent avoir été offertes à une divinité liée à la maternité, etc. Sur ce dernier point, il y a, en outre, une autre question : un sanctuaire donné est-il nécessairement attribué à une divinité «maternelle » (normalement identifiée avec Déméter, conjointement avec sa fille Korè) parce qu'on a trouvé in loco des kourotrophoi et des kourophoroi, ou bien trouve-t-on dans le sanctuaire 
donné des kourotrophoi et des kourophoroi parce que le sanctuaire est dédié à une divinité «maternelle»?

Malgré ces questions qui demeurent ouvertes, on peut proposer quelques données concrètes : la continuité, la répétitivité, le nombre élevé et l'homogénéité de certaines statuettes avec les susdites caractéristiques se trouvent en Sicile et pas ailleurs. Compte tenu de ces éléments, faibles mais concrets, on peut tirer des conclusions, même avec les limites déjà soulignées.

Les éléments recueillis pourraient faire supposer l'existence sur l'île de cultes pour des divinités kourotrophiques et chtoniennes, qui ne peuvent pas être mieux identifiées, dont les origines et les caractéristiques les plus particulières doivent être probablement recherchées dans le monde indigène. On ne trouve pas convaincante l'hypothèse d'une seule divinité commune à la Sicile entière, même avec des nuances différentes.

On pourrait se demander si les origines de ces cultes doivent être recherchées dans les mondes sicule ou bien sicane. En raison de la distribution géographique des pièces, on pourrait supposer qu'il s'agit plutôt du monde sicane, et peut-être en partie dans le monde proche-oriental. La côte ionienne et les extrémités orientale et méridionale sont les zones avec le nombre le plus petit de statuettes (mais il ne faut pas oublier qu'il s'agit de zones très urbanisées, par conséquent cette donnée pourrait être légèrement faussée). Mégara Hyblaea serait aussi, en ce cas, une exception.

Les pièces cataloguées semblent suggérer l'existence d'une sensibilité différente par rapport à celle des Grecs, qui a poussé à représenter des gestes plus naturels, des corps moins idéalisés, un lien plus explicite, « visuel » (mais pas nécessairement plus fort) entre femme (pas nécessairement plus la mère, mais pourtant une mère, si elle est en train d'allaiter) et enfant.

L'aspect "maternel », en plus de "chtonien", des divinités féminines locales serait confirmé par la découverte de statuettes d'enfants et de femmes enceintes, à côté de kourotrophoi et kourophoroi.

En partant des nombreuses mères, expression vraisemblablement de croyances de populations non grecques, on est arrivé à la théorisation d'une seule "Grande Mère » méditerranéenne pour la Sicile. À ce propos, il est très intéressant d'observer qu'à l'époque préhistorique le culte de la soi-disant «Venus » steatopyges en Sicile n'est pas si présent. Il y a des statuettes gynomorphes de plusieurs types, mais, à notre avis, elles ne témoignent pas d'une dévotion particulière pour une ou plusieurs Venus ou «Déesse Mère ». On ne trouve pas, par exemple, les célèbres statuettes stéatopyges présentes dans des différents zones d'Europe, d'Afrique et d'Asie, mais aussi dans la voisine Malte ${ }^{56}$ ou dans la (relativement) voisine Sardaigne pre-nuragique, où existait une riche production de ces statuettes à partir du Néolithique jusqu'à l'Âge du Cuivre ${ }^{57}$ : rien de comparable n'est attesté en Sicile.

\section{Conclusions}

On est parti de la pretendue "Grande Mère ", dont on a mis en discussion aussi bien l'existence en général que l'existence en Sicile. Mythe moderne, comme celui qui fait de la Sicile l'île des deux déesses (souvent dites chtoniennes), la « Grande Mère sicilienne » ne trouve pas de fondement dans la documentation en notre possession. On a essayé de réviser ce mythe surtout en analysant la documentation archéologique, en particulier le 
nombre très élevé de statuettes de kourotrophoi et kourophoroi présentes en Sicile (mais pas dans la Grèce continentale). Sur la base des données recueillies, on peut affirmer que la présence massive de ces statuettes ne prouve pas l'existence sur l'île d'un culte « spécial » adressé à une seule divinité «maternelle » qui, à partir d'un certain moment, aurait été appelée avec des noms grecs (principalement Déméter). Néanmoins, cette présence pourrait indiquer l'existence de plusieurs cultes locaux adressés aux divinités kourotrophiques (liées aux différents aspects de la maternité entendue en sens large) et chtoniennes (liées au monde des morts et à la terre, entendus soit comme source de la vie soit comme lieu de mort) qui, à partir d'un certain moment, furent désignées avec des noms grecs (par exemple Déméter et Korè). Ces divinités, on les appella tout simplement «mères ".

47 À partir de l'époque préhistorique, in primis, on a vérifié qu'en Sicile on ne trouvait pas cette abondance de statuettes stéatopyges présentes dans d'autres zones du bassin méditerranéen à la même époque (ce détail pourrait faire réfléchir ceux qui affirment, avec une inébranlable conviction, que la " Grande Mère » a existé en Sicile).

Bien que pour la plupart de pièces il soit difficile, sinon impossible, de déterminer le contexte de la découverte et la datation, nous sommes parvenue à reconstruire un espace temporel (fin $\mathrm{VII}^{\mathrm{e}}-\mathrm{IV}^{\mathrm{e}}$ siècles av. J.-C., avec une concentration très forte entre les $\mathrm{VI}^{\mathrm{e}}$ et $\mathrm{V}^{\mathrm{e}}$ siècle av.J.-C.) et les zones géographiques où se trouve la majorité des statuettes (dans l'ordre : Sélinonte, Gela, Camarine).

49 La plupart provient d'espaces consacrés liés au culte de Déméter et Korè ou de prétendues "divinités chtoniennes", mais dans les deux cas il s'agit souvent d'attributions discutables : l'attribution sur l'île d'un nombre si élevé de sanctuaires à Déméter et Korè d'un côté, et la définition très ambiguë de divinités chtoniennes de l'autre ont, en effet, favorisé des conclusions arbitraires de la part de savants modernes. Une révision au cas par cas serait nécessaire et souhaitable.

50 La documentation recueillie et analysée nous permet, néanmoins, de proposer quelques réflexions conclusives. Avec précaution, on peut affirmer que la continuité, la répétitivité, la quantité et l'homogénéité d'un nombre si significatif de kourophoroi, mais surtout de kourotrophoi, sont typiques de la seule Sicile à cette époque ${ }^{58}$. On peut alors supposer que, en Sicile, existaient plusieurs cultes adressés à des divinités kourotrophiques et chtoniennes (selon la définition qu'on vient de proposer), qu'on ne peut pas mieux définir, dont les caractéristiques les plus spécifiques pourraient être cherchées dans le monde non Grec (peut-être sicane, mais les éléments distinctifs entre les cultures sicule et sicane sont très évanescents). Il ne nous semble pas vraisemblable de parler d'une seule divinité commune - même avec des nuances - pour l'île entière. On pourrait expliquer de cette façon la différence documentaire entre Grèce continentale et Sicile. Pour ce qui concerne l'iconographie, les influences proche-orientales visibles dans certains exemplaires de production vraisemblablement locale et de datation surtout archaïque pourraient orienter la recherche en direction de contacts sans intermédiaires entre la Sicile et l'Asie Mineure. La prédominance d'une divinité féminine sur l'île pourrait avoir son origine, au moins pour une part, dans un rapport privilégié entre la Sicile et le Proche-Orient ${ }^{59}$. 


\section{BIBLIOGRAPHIE}

Adamesteanu D. (1958), « Butera : Piano della Fiera, Consi e Fontana Calda », Monumenti Antichi 44 , p. 205-672.

Adriani A. (1939), Sculture in tufo, Alexandrie (Égypte).

Annibaletto M. (2006), « Scenari sacri di epoca classica : analisi territoriale dei santuari greci nella cuspide sud-orientale della Sicilia », dans Morandi Bonacossi D. et al. éd., Tra Oriente e Occidente. Studi in onore di Elena Di Filippo Balestrazzi, Padoue, p. 117-133.

Antonetti C. et De Vido S. (2006), «Cittadini, non cittadini e stranieri nei santuari della Malophoros e del Meilichios di Selinunte ", dans Naso A. éd., Stranieri e non cittadini nei santuari greci. Atti del convegno internazionale, Florence, p. 410-451.

Asheri D. (1980), « La colonizzazione greca », dans Gabba E. et Vallet G. éd., La Sicilia antica, vol. I. 1, Naples, p. 89-142.

Banti L. (1941), « Divinità femminili a Creta nel tardo Minoico III », SMSR 17, p. 17-36.

Besques S. (1954), Catalogue raisonné des figurines et reliefs en terre-cuite grecs étrusques et romains. Époques préhellénique, géométrique, archaïque et classique, Paris.

Bonfante L. (1986), « Votive Terracotta Figure of Mothers and Children », dans Swaddling J. éd., Italian Iron Age Artefacts in the British Museum. Papers of the Sixth British Museum Classical Colloquium, London, 10-11 December 1982, Londres, p. 195-201.

- (1989), « Iconografia delle madri: Etruria e Italia antica », dans Rallo A. éd., Le donne in Etruria, Rome, p. 85-106.

- (1997), « Nursing Mothers in Classical Art », dans Koloski A.O. et Lyons C.L. éd., Naked Truths. Women, Sexuality, and Gender in Classical Art and Archaeology, Londres-New York, p. 174-196.

- (1994-1995), « La mère des dieux et Bachofen en Grèce ancienne », Mètis IX-X, p. 293-297.

Borgeaud Ph. et al. éd. (1999), La mythologie du matriarcat. L'atelier de Johann Jakob Bachofen, Genève.

- (2002), « La madre degli dei o il potere di una straniera ancestrale », dans Marino S., Montepaone C. et Tortorelli Ghidini M. éd., Il potere invisibile. Figure del femminile tra mito e storia. Studi in memoria di Maria Luisa Silvestre, Naples, p. 77-94.

Börker-Klähn J. (2003), « Tumulus D von Bayındır bei Elmalı als historischer Spiegel », dans Giorgieri M. et al. éd., Licia e Lidia prima dell'Ellenizzazione, Atti del convegno internazionale, Roma 11-12 ottobre 1999, Rome, p. 69-105.

Brelich A. (1964-1965), « La religione greca in Sicilia », Kokalos 10-11, p. 35-62.

Charbonneaux J., Martin R. et Villard F. (1995), La Grecia arcaica (620-480 a.C.), Milan (éd. or. Grèce archaïque, Paris, 1968).

Chirassi Colombo I. (1975), « Problemi per lo studio dei fatti religiosi della preistoria », dans Valcamonica Symposium '72. Actes du symposium international sur les religions de la préhistoire, Capodimonte, p. 567-581. 
- (2008), « Biografia di una dea. Demeter », dans Di Stefano C.A. éd., Demetra. La divinità, i santuari, il culto, la leggenda. Atti del 1. congresso internazionale, Enna, 1-4 luglio 2004, Pise-Rome, p. 15-23.

Ciaceri E. (1911), Culti e miti nella storia dell'antica Sicilia, Catane.

Curbera J. B. (1999), « Defixiones », dans Gulletta M.I. éd., Sicilia epigraphica. Atti del Convegno di Studi, Erice 15-18 ottobre 1998, Pise, p. 159-186.

Cusumano N. (1997), « La "religione mediterranea" un problema storico-religioso tra storia e ideologia », Mythos 9, p. 31-49.

- (2005), « "Una storiografia a rovescio”. A. Brelich e la religione greca in Sicilia ", dans Lancellotti M.G. et Xella P. éd., Angelo Brelich e la storia delle religioni : problemi e prospettive. Atti del Convegno di Roma, 3-4 Dicembre 2002, Vérone, p. 83-106.

- (2008), « Culti e miti », Kokalos 48-49, p. 442-462.

- (2009), « Mots pour dire les mots. Interactions, acculturations et relations interculturelles dans la Sicile antique ( $\mathrm{v}^{\mathrm{er}}-\mathrm{I}^{\mathrm{er}}$ siècle avant J.-C.) », Pallas 79, p. 41-63.

De Miro E. (1990-1991), « Eugenio Manni e l'archeologia siciliana », Kokalos 36-37, p. 33-41.

Di Stefano C.A. (2008), Demetra. La divinità, i santuari, il culto, la leggenda. Atti del 1. congresso internazionale, Enna, 1-4 luglio 2004, Pise-Rome.

- (2008), « Demetra a Camarina. Note di topografia. Revisioni e novità », dans Di Stefano C.A. (2008), p. 261-271.

Di Vita A. (2008), « Conclusioni. Demetra. La divinità, i santuari, il culto, la leggenda », dans Di Stefano C.A. (2008), p. 293-296.

Dodds C.A. (1997), I Greci e l'irrazionale, Milan (éd. or. The Greeks and the Irrational, Berkeley-Los Angeles 1951).

Ducaté-Paarmann S. (2003), "Les courotrophes », dans Gourevitch D., Moirin A. et Rouquet N. éd., Maternité et petite enfance dans l'antiquité romaine. Catalogue de l'exposition, Bouges, Museum d'histoire naturelle 6 novembre 2003-28 mars 2004, Bourges, p. 48-50.

- (2004), Images de la femme à l'enfant. Offrandes et cultes dans les sanctuaires d'Italie centrale et méridionale (Sicile, Grande Grèce, Campanie, Étrurie, Latium) : fin du VII ${ }^{\mathrm{e}}$ siècle - fin du II ${ }^{\mathrm{e}}$ siècle avant J.-C. , thèse de Doctorat, Paris.

Ekroth G. (2002), The sacrificial Rituals of Greek Hero-Cults in the Archaic to the Early Hellenistic Periods, Liège.

Fairbanks A. (1900), « The Chthonic Gods of Greek Religion », AJPh 21, p. 241-259.

Finley M.I. (1981), Uso e abuso della storia, Turin (éd. or. The Use and Abuse of History, Londres, 1975).

Giannitrapani E. (1997), « Sicilia e Malta durante il neolitico », dans Tusa S. éd., Prima Sicilia. Alle origini della società siciliana. Catalogo della mostra, Albergo dei poveri, Palermo, 18 ottobre-22 dicembre 1997, Palerme, p. 429-444.

Georgoudi S. (1997), Bachofen, il matriarcato e il mondo antico : riflessioni sulla creazione di un mito, dans Duby G., Perrot M. et Schmitt Pantel P. éd., Storia delle donne. L'Antichità, Rome-Bari (éd. or. Histoire des femmes en Occident. L'Antiquité, Paris, 1991), p. 518-536.

Giammellaro P. (2008), « Religione e religioni della Sicilia antica nell'opera di Emmanuele Ciaceri », SMSR 74, p. 49-73.

- (2012), « Biagio Pace e la Sicilia antica », StudStor 53, p. 391-420. 
Gras M. et al. (2004), La ville archaïque. L'espace urbain d'une cité grecque de Sicile orientale, Rome.

Hadzisteliou Price T. (1978), Kourotrophos. Cult and Representations of the Greek Nursing Deities, Leyde.

Hinz V. (1999), Der Kult von Demeter und Kore auf Sizilien und in der Magna Graecia, Wiesbaden.

Langlotz-Hirmer E. (1963), Die Kunst der Westgriechen in Sizilien und Unteritalien, Munich.

La Rosa V. (1989), « Le popolazioni della Sicilia. Sicani, Siculi, Elimi », dans Pugliese Carratelli G. éd., Italia omnium terrarum parens, Milan, p. 3-110.

- (1996), «L'incontro dei coloni greci con le genti anelleniche della Sicilia », dans Pugliese Carratelli G. éd., I Greci in Occidente. Venezia, Palazzo Grassi, 24 marzo-8 dicembre 1996, Ministero per $i$ beni culturali e ambientali, Milan, p. 523-532.

Le Dinahet M.-T. (1984), « Sanctuaires chthoniens de Sicile de l'époque archaïque à l'époque classique », dans Temples et sanctuaires. Séminaire de recherche 1981-1983 sous la direction de G. Roux, Lyon, p. 137-152.

Loraux N. (1997), « Che cos’è una dea ? », dans Duby G., Perrot M. et Schmitt Pantel P. éd., Storia delle donne. L'Antichità, Rome-Bari (éd. or. Histoire des femmes en Occident. L'Antiquité, Paris, 1991), p. 13-55.

Lo Schiavo F. (2009), « I rapporti fra Sicilia e Sardegna nel II millennio a.C. », dans Ampolo C. éd., Immagine e immagini della Sicilia e di altre isole del Mediterraneo antico. Atti di un convegno tenuto a Erice nel 2006, Pise, p. 401-419.

Manni E. (1963), Sicilia pagana, Palerme.

- (1975), « Da Megara Iblea a Selinunte : le divinità », Kokalos 21, p. 174-195.

Martinez J.-L. (2000), La dame d'Auxerre, Paris.

Martorana G. (2003), Prefazione alla ristampa di E. Manni, Sicilia Pagana, Palerme, p. 9-16.

Momigliano A. (1987), «Johan Jacob Bachofen: From Roman History to Matriarchy », dans Ottavo contributo alla storia degli studi classici e del mondo antico, Rome, p. 91-106.

Orlandini P. (1962), «L'espansione di Gela nella Sicilia centro-orientale », Kokalos 8, p. 69-121.

- (1966), « Gela : nuove scoperte del Thesmophorion di Bitalemi », Kokalos 13, p. 177 ss.

- (1968-1969), « Diffusione del culto di Demetra e Kore in Sicilia », Kokalos 14-15, p. 334-338.

- (2008), « Demetra a Gela », dans Di Stefano C.A. (2008), p. 173-186.

Orsi P. (1897), « Siracusa », NSA, p. 471-504.

Pace B. (1935-1949), Arte e civiltà della Sicilia antica, Rome-Naples-Città di Castello.

Panvini R. et Sole L. (2009), La Sicilia in età arcaica. Dalle apoikiai al 480 a.C., Palerme.

Parisi Presicce C. (1986), « La struttura », Sicilia Antica 19, p. 40-53.

Patané R.P.A. (2008), « Demetra a Centuripe », dans Di Stefano C.A. (2008), p. 255-260.

Pedrucci G. (2007), « Presenze metroache in area elima ? ", Hormos 9, p. 437-441.

- (2009), Cibele frigia e la Sicilia. I santuari rupestri nel culto della dea, Rome.

- (2013a), L'isola delle « madri ». Una rilettura della documentazione archeologica di donne con bambini in Sicilia, Rome.

- (2013b), L'allattamento nella Grecia di epoca arcaica e classica, Rome. 
Pugliese Carratelli G. (1985), Ichnussa. La Sardegna dalle origini all'età classica, Milan (1 ère éd. 1981).

- (2000), «Il re Hyblon e le Hyblai di Sicilia », dans Di Vita A., Un ponte fra l'Italia e la Grecia. Atti del simposio in onore di Antonino Di Vita, Ragusa, 13-15 febbraio 1998, Padoue, p. 125-127.

Rizza G. et De Miro E. (1985), « Le arti figurative dalle origini al v secolo a.C. », dans Pugliese Carratelli G. éd., Sikanie. Storia e civiltà della Sicilia greca, Milan, p. 125-242.

Scullion S. (1994), « Olympian and Chthonian », CA 13, p. 75-119.

Sfameni Gasparro G. (1973), I culti orientali in Sicilia, Leyde.

Spatafora F. (2008), « Entella : il Thesmophorion di Contrada Petraro », dans Di Stefano C.A. (2008), p. 273-284.

Sposito A. (2008), « Architettura e rito nel santuario delle divinità ctonie a Morgantina », dans Di Stefano C.A. (2008), p. 223-233.

Trotta F. (2000), « I culti della Sicilia : tra Greci e Iblei », dans Di Vita A., Un ponte fra l'Italia e la Grecia. Atti del simposio in onore di Antonino Di Vita, Ragusa, 13-15 febbraio 1998, Padoue, p. 155-160.

Veronese F. (2006a), Lo spazio e la dimensione del sacro. Santuari greci e territorio nella Sicilia arcaica, Padoue.

- (2006b), « "Figure d'acqua” nei santuari della Sicilia greca. Significato e iconografia della figura dell'idrofora ", dans Colpo I., Favaretto I. et Ghedini F. éd., Iconografia 2005. Immagini e immaginari dall'antichità classica al mondo moderno. Atti del Convegno internazionale, Venezia, Istituto veneto di scienze lettere e arti, 26-28 gennaio 2005, Rome, p. 419-424.

Zuntz G. (1971), Persephone. Three Essays on Religion and Thought in Magna Graecia, Oxford.

\section{NOTES}

1. J'aimerais adresser des remerciements particuliers au Prof. Jean-Baptiste Bonnard et à la Prof. Florence Gherchanoc pour l'aide patiente dans la préparation de cet article en français. Je voudrais également adresser des remerciements à la Prof. Véronique Dasen pour avoir toujours cru en mon travail.

2. La théorie selon laquelle toutes les figures féminines du panthéon grec représentent les transformations successives d'une seule déesse primordiale, ancestrale, universelle, définie comme la « Grande Déesse » ou « Grande Mère » a pour fondateur Johann Jakob Bachofen, auteur du célèbre Das Mutterrecht. Eine Untersuchung über die Gynaikokratie der Alten Welt nach ihrer Religiösen Rechtlichen und Natur, Stuttgart, 1861. La spécificité des différentes déesses ne pouvant cependant pas être mise en question, la théorie de Bachofen peut être considérée comme dépassée. Cette thématique est largement débattue par Borgeaud 1999; voir aussi Momigliano 1987, Borgeaud 1994-1995, Georgoudi 1997, Borgeaud 2002, p. 87-91. Il faut préciser que Bachofen a subi une censure incroyable, autant dans le milieu universitaire qu'ecclésiastique. Le juriste suisse est d'habitude mentionné par des marxistes, des féministes et des psychanalystes, qui souvent ne l'ont pas réellement lu : Georgoudi (1997, p. 525) parle d'une « vulgata bachofaniana, semplicistica e piuttosto sbagliata ", souvent utilisée au lieu de l'original (cf. Cusumano 1997, p. 40). Il faut ajouter que le mot «matriarcat » n'apparaît jamais dans le travail de Bachofen, même si ce dernier est considéré comme l'inventeur; celui-ci parle plutôt du droit maternel (reconnaissance exclusive de la filiation matrilinéaire combinée avec le droit de succession réservé aux filles) et de gynécocratie (la supériorité de la femme autant dans la famille que dans la société). Le terme " matriarcat », créé à la fin du XIX siècle sur le modèle du terme "patriarcat 
", fond les deux aspects et, par conséquent, a connu une grande diffusion. L'idée principale de Bachofen est l'inclination naturelle des femmes au surnaturel, au divin, à l'irrationnel, d'où l'importance extraordinaire de l'image archétypale de la "Grande Déesse », "Grande Mère », " Terre-Mère », figure dominante du « royaume maternel », avec laquelle toutes les figures divines du monde ancien seraient identifiées.

3. Banti 1941, p. 36.

4. Finley 1981, p. 126.

5. Chirassi Colombo 1975, p. 579. Cf. Chirassi Colombo 2008, p. 18.

6. Hérodote, VII, 153 ; Pindare, Olympiques, VI, 94 sq. ; Diodore, V, 2 ; Cicéron, Verrines, act. II, IV, 106.

7. Pedrucci 2013a, p. 67-71 et 209-226.

8. Brelich 1964-1965, p. 38, 40 : « quel presunto culto indigeno che sarebbe alla base dell'immensa diffusione del culto di Demeter e della Kore, uno dei dogmi dei sostenitori dell'importanza del sostrato ", observe qu'il faudrait se demander "soltanto se i culti di Demeter non fossero altrettanto diffusi anche in Grecia: e troveremo, per risposta, che si nessuna festa, come i Thesmophoria, si può dimostrare la diffusione, oltre ogni barriera statale, dialettale e geografica, in tutta la Grecia ». À côté de savants comme Eugenio Manni et Giuseppe Martorana (voir n. 24), qui ont nourri et enrichi cet "axiome », ce « dogme », plus récemment on peut renvoyer aux thèses de De Vita (2008) et Curbera (1997, p. 397, 407), selon lequel « indeed, archaeological and numismatic evidence abundantly confirms the literary sources ». L'apparente absence de noms théophoriques dans les inscriptions siciliennes peut s'expliquer par la nature des «strongly plebeian and feminine » du culte de Déméter sur l'île. Sur ce dernier point, voir n. 16, Curbera se réfère à certaines inscriptions tardives (les plus anciennes sont datées des $\mathrm{IV}^{\mathrm{e}}$-III ${ }^{\mathrm{e}}$ siècle av. J.-C., les plus récentes des $\mathrm{I}^{\mathrm{e}}-\mathrm{II}^{\mathrm{e}}$ ap. J.-C.), en supposant que l'adjectif «chthonien » désigne en Sicile exclusivement Déméter et Korè ; pour ces raisons, nous exprimons des doutes sur cet article.

9. Selon Hinz (1998), seules les femmes aux porcelets et les bustes sont typiques du culte de Déméter et Korè. À notre avis, les bustes indiquent exclusivement l'existence d'un culte démétriaque. Seules les statuettes au porcelet peuvent être vraisemblablement considérées comme distinctives, mais leur découverte occasionnelle, si elle n'est pas confirmée par d'autres éléments, ne peut pas témoigner avec certitude de la présence des deux déesses : c'est, en fait, un type d'ex-voto qui a connu ce que nous appellerions aujourd'hui une production de masse et qui, par conséquent, facilement accessible et probablement peu cher, peut avoir été offert dans une grande variété de contextes comme une manifestation de pietas « générique ». En cas de présence d'offrandes au porcelet, on ne peut pas exclure l'existence d'un culte de Déméter et Korè, compte tenu du rôle particulier du porcelet dans ce culte, mais on ne peut pas l'affirmer sans un examen exhaustif et minutieux des données disponibles.

10. Adjectif très utilisé par les historiens des religions et les archéologues, il est, en raison précisément de cette utilisation abondante, souvent cause de confusion et d'approximation. Les Grecs, cependant, avaient déjà les idées peu claires sur ce mot. Voici plus d'un siècle, un débat très aminé a eu lieu sur la définition de « chtonien " par opposition à « olympien ». Fairbanks (1900) a proposé d'utiliser l'adjectif « chtonien » soit pour indiquer à la fois les dieux liés à l'âme des morts et les héros, soit pour désigner des rites propitiatoires et purificatoires associés à ces entités et aux morts. Sur la base de cette classification, Hadès, Perséphone, Gaia, Hécate et les Furies sont des divinités chtoniennes, mais aussi Zeus, Déméter et Hermès, dieux typiquement olympiens. Sur des bases non conformes à celles des auteurs anciens, les chercheurs modernes ont tendance à mettre au centre du débat le rituel plutôt que le "caractère " des dieux, qui présente de nombreuses nuances et qui ne semble pas déterminer le choix du rituel. Les limites d'une définition de ce type appliqué aux entités divines individuelles sont évidentes, comme il est démontré justement par le cas de Déméter. La question, en fait, est particulièrement importante en Sicile, où les chercheurs ont reconnu l'existence de nombreuses déesses chtoniennes, souvent 
identifiées avec Déméter et Korè. On utilise l'adjectif «chtonien» essentiellement pour caractériser les cultes adressés à des divinités associées, à différents titres, avec le monde des morts et avec la terre (entendue comme le lieu de repos des défunts, mais aussi, si elle est cultivée, comme source de vie); on ne l'utilise pas en opposition à l'adjectif "olympien » et comme synonyme de « démétriaque ». Voir Fairbanks 1900, Le Dinahet 1984, Scullion 1994 (avec bibliographie précédente), Ekroth 2002 ; Pedrucci 2013a, p. 73-75.

11. Brelich 1964-1965, p. 50.

12. L'expansion et l'importance accrue du culte de Déméter et Korè à la fin de l'époque archaïque et le possible lien avec les Deinoménides et leur hypothétique politique religieuse; les aspects particuliers du culte de Déméter et Korè en Sicile; la cohérence entre les différents types de pièces archéologiques et l'attribution des lieux de culte; les attributions faites sur la base d'un seul attribut ; l'identité exacte des « divinités chtoniennes » ... Voir Pedrucci 2013a, p. 209-225.

13. Sur la base de données recueillies par Francesca Veronese, on a 52 attestations de Déméter et 49 de Korè contre 10 d'Athéna, 8 de Héra, 7 d'Aphrodite, 4 d'Artémis et 3 des nymphes. Selon Veronese (2006a, p. 546, fig. 12.9), «questo aspetto meriterebbe forse una maggiore attenzione poiché, pur senza voler negare l'importanza dei culti ctonî anche alla luce dell'utilizzo politico che ne è stato fatto a partire dal v sec. Con i Dinomenidi, la sperequazione delle attestazioni tra il complesso dei culti ctonî e tutti gli altri è decisamente eccessiva e lascia adito al dubbio che l'interpretazione del dato possa non corrispondere alla realtà. 0 , per meglio dire, che il dato archeologico vada interpretato diversamente. L'identificazione della titolarità del culto è infatti il più delle volte basata sul solo materiale votivo (statuette, maschere) la cui produzione "seriale" [...] non permetteva una facile differenziazione tra le figure divine. Se dunque è difficile, in molti casi, operare un'effettiva distinzione, va sottolineato che non è forse neppure corretto identificare sistematicamente una cultualità specifica (ctonia) sulla base di materiali dai connotati generici che, proprio per questo, potevano facilmente configurarsi come ex-voto per altre divinità. Un problema, quest'ultimo, la cui complessità richiederebbe una trattazione in termini più specifici ». Cf. Annibaletto 2006, p. 129, voir note 15.

14. Pedrucci 2013a, p. 28-71.

15. Manni 1963, p. 63.

16. Orlandini 1966. Cf. Orlandini 1968-1969, Orlandini 2008, p. 174. Il est évident que certains chercheurs modernes ont voulu suivre aveuglement les auteurs anciens, comme par exemple Orlandini (1968-1969, p. 334) : «Diceva Cicerone nelle Verrine che tutta la Sicilia sembrava essere consacrata a Demetra e Kore e che ciò non risultava soltanto dalle testimonianze letterarie e dai monumenti, ma anche da una persuasione intima dei Sicelioti così profonda da parere quasi innata. Di fatto le scoperte archeologiche confermano tale diffusione e popolarità del culto di Demetra e Kore in Sicilia». On retrouve les mêmes idées exprimées, par exemple, par Hadzisteliou Price (1978, p. 184), qui, à la page 186, parle aussi de l'« anonymous Great Mothers, often called The Chthonic deities or Meteres, were venerated in Sicily ». Cf. Curbera 1997, p. 397 : «the whole island was said to be sacred to Demeter and Kore [...] indeed, archaeological and numismatic evidence abundantly confirms the literary sources ». Et encore plus récemment, la même croyance est manifeste dans la plupart des interventions au congrès de 2004 Demetra. La divinità, i santuari, il culto, la leggenda (Di Stefano 2008), à la fin duquel Di Vita (2008, p. 295), affirma : "Dagli interventi di oggi è apparso chiaro che tutta la Sicilia, da Entella a Siracusa, era votata a Demetra » (selon Spatafora 2008, p. 284, le culte de Déméter et Korè serait attesté jusqu'à la zone élyme). Très intéressante est l'opinion de Patané (2008, p. 256), selon lequel des « elementi del culto di Demetra sembrano passati alla Madonna della Visitazione ». Cf. n. 8.

17. Cusumano 2005. Cf. Cusumano 2009, p. 42-47.

18. Manni 1963.

19. «Substrat» est un terme privilégié par les linguistes, qui l'utilisent pour indiquer une situation de persistance culturelle à travers deux ou plusieurs stratifications linguistiques, voir 
Cusumano 1997. En ce qui concerne en particulier le substrat méditerranéen, Cusumano souligne la fragilité du cadre unitaire de la Méditerranée : son histoire ne se compose pas uniquement de circulations, d'échanges et d'interactions, mais aussi de discontinuités et de fractures.

20. Asheri 1980, p. 138 : les Grecs «dovettero assuefarsi all'acqua bastarda di Aretusa: si sicilianizzarono ». Il y a, bien sûr, les partisans du processus inverse, c'est-à-dire de la grécisation des populations locales, comme, après Brelich, Zuntz 1971. Voir Giammellaro 2008, Pedrucci 2013a, p. 5-71 (aussi pour la bibliographie sur l'histoire des études).

21. Ciaceri 1911, p. 2.

22. Pace 1935-1949. Voir Giammellaro 2012.

23. Voir Cusumano (2005, p. 86 sq.) qui analyse en particulier l'œuvre d'Uberto Pestalozza.

24. Sur la question, voir aussi Martorana 2003, p. 10 : «allora, come oggi, si deve parlare di una civiltà compatta mediterranea, ovvero di una koiné di natura agraria che in tutta la sua implicanza riportava alla Grande Madre ed al suo paredro? Sicilia pagana ripropone siffatto interrogativo. Tuttavia Eugenio Manni ammetteva l'incontro-scontro tra Indoeuropei e Mediterranei che diede vita a incomprensioni, almeno da parte indoeuropea ».

25. « La questione della continuità si lega strettamente [...] a quella della costruzione dell'identità e della ricerca di legittimazione attraverso la memoria (più o meno storica) del passato. Essa richiede una riflessione sull'ambigua nozione di sopravvivenza [...] Non ci sono sopravvivenze o relitti tout court, ma funzioni simboliche; non ci sono elementi culturali che sopravvivono, ma elementi - o meglio reti di elementi - che agiscono con funzioni nuove in contesti differenti, senza con ciò negare troppo rigidamente la possibilità di residui imperfettamente ricollocati. Per tale ragione ciò che ci appare come persistenza deve essere capito in seno alla cultura in cui si esprime, perché ne costituisce un dato vitale »: Cusumano 2008, p. 457 sq.

26. Dodds 1997, p. 36 sq. À propos de l'œuvre de Dodds, voir Cusumano 2005, p. 93 sq.

27. Bonfante 1986 ; Bonfante 1989 ; Bonfante 1997. Voir Pedrucci 2013a, p. 119-123.

28. Pedrucci 2013a, p. 75.

29. La variante la plus connue est celle de la femme à l'enfant sur son épaule gauche. Le geste est effectivement attesté d'une manière extraordinaire en Sicile et, en particulier, à Bitalemi (voir infra), toutefois il n'est pas rare dans d'autres parties du monde antique. Voir, pour quelques exemplaires de la Grèce, par exemple Hadzisteliou Price 1978, fig. 3. Le geste se trouve également dans les représentations hellénistiques de nourrices âgées, voir Pfisterer-Haas 1989, Abb. 46-51. Il faut aussi signaler un exemplaire de Bayındır près d'Elmalı, où, dans la sépulture d'une femme, a été retrouvée une statuette de femme richement vêtue d'un polos, avec un enfant nu sur l'épaule gauche et un autre enfant, lui aussi habillé avec luxe, tenu par la main. La statuette est datée environ du début $d u \mathrm{VII}^{\mathrm{e}}$ siècle av. J.-C. et révèle des influences phrygiennes, voir Börker-Klähn 2003, p. 77 sq., Abb. 4. Pour les possibles contacts entre la Phrygie et la Sicile avant la colonisation grecque, voir Pedrucci 2009.

30. Le terme même de kourophoros n'est jamais attesté dans la langue grecque; il nous semble nécessaire (cf. Parisi Presicce 1986) de distinguer entre les statuettes de femmes assises qui allaitent un enfant (kourotrophoi) et les statuettes de femmes qui portent un enfant (kourophoroi). Nous avons également préféré utiliser la minuscule (cf. n. 54), en absence d'éléments suffisants pour déterminer la nature divine ou humaine des figures représentées. Pour kourotrophos et les significations du verbe trephô, voir Pedrucci 2013a, p. 71-73.

31. Pedrucci 2013a, p. 131-208, en particulier pour la bibliographie.

32. Pour la position par rapport à la colonie, voir Veronese 2006a. Pour les sanctuaires extraurbains, voir Pedrucci 2013a, p. 100-103.

33. Veronese 2006a, p. 410. Butera a été le premier centre indigène à entrer en contact avec Gela, probablement au cours de la première moitié du viI ${ }^{\mathrm{e}}$ siècle av. J.-C., et dès cette époque il présente un niveau significatif d'hellénisation. Le culte dans le sanctuaire de Fontana Calda fut extraordinairement de longue durée : à partir de la seconde moitié du VII ${ }^{\mathrm{e}}$ siècle av. J.-C. jusqu'à 
la période byzantine. Sur la base des ex-voto retrouvés et des caractéristiques environnementales (proximité des sources, forte odeur d'iode, présence de grottes), on croit que le lieu pouvait être un endroit pour un culte de Déméter et Korè ou le centre d'un culte des eaux (dédié à une nymphe ?). On y a trouvé deux terres cuites de femmes enceintes et une kourophoros à l'enfant sur l'épaule gauche du $\mathrm{v}^{\mathrm{e}}$ siècle av. J.-C. La datation des nombreuses et très fragmentaires terres cuites provenant de la stipe votive est entre la seconde moitié $\mathrm{du} \mathrm{VI}^{\mathrm{e}}$ et le $\mathrm{II}^{\mathrm{e}}$ siècles av. J.-C. La production, au moins en partie, est locale, mais les motifs sont bien connus sur toute l'île, surtout dans le territoire de Gela. Il peut être intéressant de noter que, encore au milieu du siècle dernier, la source de Fontana Calda était fréquentée par des femmes sur le point d'accoucher pour faire des ablutions avec l'eau. Les ex-voto des femmes enceintes trouvés dans le stipe ont fait supposer qu'un rite similaire avait ses racines dans une époque ancienne; toutefois, des affirmations de ce type, relatives à des continuités plus que bimillénaires requièrent une grande prudence. Voir Adamesteanu 1958, p. 627, fig. 47 ; Veronese 2006b, p. 422, fig. 4b, c. Des statuettes de femmes enceintes ont été trouvées aussi dans les sanctuaires de Carrubazza et Capodarso. Pour la représentation des femmes enceintes dans le monde grec, voir Pedrucci 2013b, p. 74-78.

34. Orlandini 1962, 110 sq. Les autres noms attestés sont Kypra, Kypara et Atita. Bien évidemment, elles ne sont pas des déesses grecques. La première et la deuxième pourraient être Cupra ombrienne, mais la troisième pourrait rappeler Cybèle et Attis, cf. Pedrucci 2007.

35. Panvini-Sole 2009, II, p. 77, VII/19 ; Pedrucci 2013a, Scheda 96.

36. Panvini-Sole 2009, II, p. 407, TA/50; Pedrucci 2013a, Scheda 97.

37. Zuntz 1971, p. 151, pl. 21d ; Hadzisteliou Price 1978, p. 22, fig. 11 ; Pedrucci 2013a, Scheda 99.

38. Il existe une variante identique, mais sans enfant, voir Besques 1954, p. 80, B560 ; Zuntz 1971, p. 11 sq., pl. 14b ; Pedrucci 2013a, Scheda 100.

39. Rizza-De Miro 1985, 174, fig. 174 sq. Cf. Hadzisteliou Price 1978, p. 31 sq., p. 182 ; Bonfante 1989, p. 87, tav. XXXV ; Bonfante 1997, p. 178 ; Pedrucci 2013a, Scheda 98. Selon Hadzisteliou Price (1978, p. 182), elle est « similar in nature to an older indigenous goddess, perhaps Hyblaia ». Dans le compte rendu des fouilles du temple B ont été signalés des fragments de deux grandes statues féminines en terre cuite du vi ${ }^{\mathrm{e}}$ siècle av. J.-C. ; voir Gras 2004, p. 333.

40. Ce dernier, d'origine inconnue, est attribué à l'école dédalo-crétoise, avec des influences égyptiennes, voir Martinez 2000. Pour d'autres statues, voir Charbonneaux-Martin-Villard 1995, p. 135 sq., p. 139.

41. Rizza-De Miro 1985, fig. 178 : statue féminine du Grammichele, fig. 181 : tête au heaume.

42. La Rosa 1989, p. 59, fig. 73 ; La Rosa 1996, p. 525 : « Il dettaglio dei seni traboccanti dalla veste non consente [...] che generici richiami alla sfera della fecondità », p. 527 ; Pedrucci 2013a, p. 172, n. 176, pour la nudité frontale.

43. Pugliese Carratelli 2000 ; Trotta 2000. Il serait possible de voir dans le terme Hybla un nom «"collegato alla sfera sacrale di una nazione anatolica" e passato dalla cultura sicana a quella sicula come dinastico "parlante", un "nome regale tratto da un centro che forse era stata capitale della dinastia sicana". Risaliremmo quindi, ancora attraverso i Sicani, a esperienze di contatto tra indigeni della Sicilia orientale e elementi egeo-anatolici che porterebbero fino alla Caria »: Trotta 2000, p. 159.

44. Ou doit-on considérer tous les ex-voto aux colombes (ou bien avec un oiseau en général) comme faisant partie du culte d'Aphrodite? On pense que non. Des statuettes aux colombes ont été découvertes dans des sanctuaires dédiés à Héra, Déméter ou d'autres divinités (voir DucatéPaarmann 2004, p. 27) ; les colombes sont aussi un attribut de Korè (voir Sposito 2008, p. 224).

45. Voir n. 10.

46. Hadzisteliou Price 1978, p. 181 sq. ; Hinz 1998 ; Veronese 2006 .

47. Hadzisteliou Price 1978, p. 181 sq.

48. Ducaté-Paarmann 2003, p. 48 sq. 
49. Les seules exceptions que nous connaissons sont des statues en tuf de Santa Maria Capua Vetere, représentant des femmes assises avec un ou plusieurs enfants sur les genoux ou dans les bras. Cependant, elles sont le produit de l'art italique, et non pas grec. La caractérisation marquée, la tendance réaliste, les traits grossiers et naifs sont plutôt typiques de l'art étrusque. Selon Adriani (1939, p.18), «si tratta di una produzione d'arte popolare, incolta, senza tradizione, e perciò più che mai contraddittoria e capricciosamente varia a seconda dell'abilità $\mathrm{e}$ della personale capacità inventiva o imitativa dello scultore ». En ce qui concerne la chronologie, on n'a pas d'éléments de comparaison avec des productions régionales déjà connues, et les données topographiques et stratigraphiques ont été irrémédiablement perdues. La majorité se date des $\mathrm{II}^{\mathrm{e}-\mathrm{I}^{\mathrm{er}}}$ siècles av. J.-C. (les traits sont grossiers, mais pas archaïques; il y a des inscriptions qui confirment la datation), mais pour certaines on peut proposer une datation entre le $\mathrm{VI}^{\mathrm{e}}$ et le $\mathrm{V}$ e siècle av. J.-C. Selon Adriani, les statues représentent des mères mortelles, chacune avec le nombre de ses enfants. Adriani lui-même, toutefois, n'exclut pas que les statues représentent des divinités, qui seraient l'expression d'un culte antique et populaire, non importé. Voir Adriani 1939 ; Langlotz-Hirmer 1963, p. 101, cat. 167 sq.

50. On peut identifier quelques éléments distinctifs de l'art indigène : la caractéristique la plus évidente est une tendance linéaire, qui est liée à la construction volumétrique, pas dans le sens grec d'une articulation organique des masses, mais des masses juxtaposées et contrastées. Comme l'a justement observé De Miro (1990-1991, p. 40), « per noi, invece, è possibile cogliere se non un'arte, una sensibilità formale indigena, con alcune caratteristiche rintracciabili nella produzione siceliota ». Voir également Pedrucci 2013a, p. 86-95, aussi pour les problématiques liées aux distinctions ethniques.

51. Dans la Sicile occidentale, à la différence de ce que l'on voit dans la partie orientale de l'île, les sanctuaires où on peut supposer la présence pacifique des différents groupes ethniques ne sont pas rares, par exemple Éryx, Monte Jato, Ségeste et Mothia ; voir Antonetti-De Vido 2006, p. 423 sq.

52. Le lien de cette figure divine avec la naissance et la mort est très évident, même si, selon Antonetti, les aspects kourotrophiques ne sont pas pertinents pour la Malophoros, à la différence de la divinité du Edificio Triolo nord, voir Antonetti-De Vido 2006, p. 434.

53. Veronese 2006a, p. 557 sq., 602, fig. 12.21.

54. En paraphrasant Loraux (1997, p. 38), il y a la Mère, les Mères, la Grande Mère, la Grande Déesse Mère, la Déesse, dans une sorte d'emphase lexicale et sémantique où «il gusto della maiuscola ha libero sfogo, in cui l'impulso dominante è quello di cancellare le differenze ", avec le résultat de rendre illusoire et vain chaque raisonnement historique. En ce qui concerne le pluriel, pour Loraux (1997, p. 28 sq.), la rencontre entre féminin et pluriel n'est pas casuelle. Voir Pedrucci 2013b, p. 130-133. Cf. n. 30.

55. L'adjectif «maternel » est utilisé ici peut-être d'une manière anachronique, puisque ce que nous entendons par maternel pourrait ne pas coïncider avec la signification que les Grecs attribuaient à ce terme. Voir Pedrucci 2013b, p. 154, n. 642.

56. Pedrucci 2013a, p. 125-39. Cf. Giannitrapani (1997, p. 443) qui, en parlant de Malte, affirme : « Sono presenti, inoltre una serie di statuette fittili ginecomorfe che richiamano esemplari noti anche nel resto del Mediterraneo, ma non in Sicilia ».

57. Pugliese Carratelli 1985, p. 21-51, fig. N48-N61, N64-N68. Pour les rapports entre Sicile et Sardaigne, voir Lo Schiavo 2009.

58. Du moins, on n'en trouve pas à l'extérieur de l'Italie antique : comme nous l'avons vu (voir n. 27), la documentation relative aux femmes et aux enfants est également très riche dans l'Italie du Sud et en Étrurie, mais nous préférons ne pas étendre la comparaison à ces territoires, puisque nous ne les avons pas encore personnellement analysés.

59. Peut-être en allant vers l'Anatolie : à notre avis un lien très fort et sans intermédiaire entre Sicile et Anatolie est évident dans le sanctuaire rupestre d'Akrai, dans certaines gravures faites 
dans la roche des Monts Hybléens et sur quelques terres cuites représentant Cybèle, voir Pedrucci 2007, Pedrucci 2009, Pedrucci 2013a, p. 104.

\section{RÉSUMÉS}

La Sicile offre une quantité surprenante de statuettes de femmes à l'enfant, parmi lesquelles on trouve en abondance un sujet très rare dans le monde ancien (surtout en Grèce continentale) : celui de la mère qui allaite. On essayera d'expliquer cette extraordinaire documentation archéologique, en relativisant les très puissants « mythes » de la «Grande Mère sicilienne » et de la Sicile comme terre consacrée à Déméter et Korè et en préférant chercher les origines des cultes liés aux terres cuites des femmes à l'enfant chez les populations non grecques, sans exclure des apports proche-orientaux.

Sicily has an impressive number of terracottas representing women with children, between them we find a very rare motif in the ancient world (above all in continental Greece) : the breastfeeding woman. In the effort to explain this extraordinary archaeological documentation, it is necessary to review the very powerful « myths » of the « Sicilian Great Mother » and of Sicily as a land completely sacred to Demeter and Kore. The origins of the cults connected with the terrecottas of women with children have to be searched for, in our opinion, in the ante-hellenic cultures, taking onto account the possibility of direct contacts with the ancient Near East.

\section{INDEX}

Mots-clés : kourotrophoi, Sicile, Proche Orient, Grande Mère, maternité divine, allaitement Keywords : Sicily, Near East, Great Mother, divine motherhood, breast-feeding

\section{AUTEUR}

\section{GIULIA PEDRUCCI}

Université de Bologne 